the studies. First, the rate of individual adverse events between the MRSA/VRE and non-MRSA/VRE patients in Martin et al are not included. Second, for rate calculation, we calculated the rate per 1,000 patient days, whereas Martin et al considered the rate per 1,000 admissions. Whether this had any influence on outcomes is unknown.

Similar to the previous study by Martin et $\mathrm{al}^{3}$ that indicated no change in the healthcare associated infection (HAI) rates of MRSA/VRE after elimination of $\mathrm{CP}$, we also reported no significant change in HAI rates in MRSA/VRE patients after eliminating $\mathrm{CP}$ in our study. ${ }^{2}$ Thus, eliminating CP for MRSA/VRE patients is not associated with increased HAI rates with MRSA/ VRE and could improve patient safety outcomes. Our observation that MRSA/VRE patients are at higher risk of noninfectious adverse events argues the need for serious consideration of eliminating CP among MRSA/VRE patients.
Financial support. No financial support was provided relevant to this article.

Conflicts of interest. All authors report no conflicts of interest relevant to this article.

\section{References}

1. Martin EM, Bryant B, Grogan TR, et al. Noninfectious hospital adverse events decline after elimination of contact precautions for MRSA and VRE. Infect Control Hosp Epidemiol 2018. Published online May 10, 2018, p. 1-9.

2. Gandra S, Barysauskas C, Mack DA, Barton B, Finberg R, Ellison RT III. Impact of contact precautions on falls, pressure ulcers and transmission of MRSA and VRE in hospitalized patients. J Hosp Infect 2014; 88:170-176.

3. Martin EM, Russell D, Rubin Z, et al. Elimination of routine contact precautions for endemic methicillin-resistant Staphylococcus aureus and vancomycin-resistant Enterococcus: a retrospective quasi-experimental study. Infect Control Hosp Epidemiol 2016; 37:1323-1330.

\title{
Collaboration for containment: Detection of OXA-23-like carbapenamase-producing Acinetobacter baumannii in Colorado
}

\author{
Heather L. Young $\mathrm{MD}^{1}$, Caroline Croyle $\mathrm{MPH}^{2}$, Sarah J. Janelle MPH, $\mathrm{CIC}^{3}$, Bryan C. Knepper MPH, MSc, $\mathrm{CIC}^{2}$, \\ Jennifer Kurtz BSN, MS ${ }^{2}$, Amber Miller MSN, CIC, CHFM ${ }^{4}$, Sara M. Reese PhD, MPH, $\mathrm{CIC}^{5}$, Kyle Schutz MSc ${ }^{3}$ and \\ Wendy M. Bamberg MD ${ }^{3}$ \\ ${ }^{1}$ Department of Medicine, Denver Health Medical Center and University of Colorado School of Medicine, Denver, Colorado, ${ }^{2}$ Department of Patient Safety and \\ Quality, Denver Health Medical Center, Denver, Colorado, ${ }^{3}$ Colorado Department of Public Health and Environment, Denver, Colorado, ${ }^{4}$ Department of Planning \\ and Construction, University of Colorado Health, Aurora, Colorado and ${ }^{5}$ Department of Quality. Swedish Medical Center, Denver, Colorado
}

To the Editor-Multidrug-resistant Acinetobacter baumannii (MDR-AB) is an aggressive pathogen often transmitted in healthcare facilities. Critically ill patients are at highest risk, particularly those with recent surgery, prolonged ventilation, and exposure to broad spectrum antibiotics. ${ }^{1}$ Containment of MDR$A B$ requires early identification and multifaceted interventions.

MDR-AB strains that are resistant to carbapenems present additional containment issues because plasmid-mediated carbapenemase production is a common resistance mechanism. ${ }^{2}$ Given its importance as an emerging antimicrobial-resistant pathogen, many public health departments, including the Denver metropolitan region in Colorado, require carbapenem-resistant Acinetobacter baumannii (CRAB) to be reported.

Between December 2017 and February 2018, Denver Health Medical Center (DHMC) detected 2 inpatients with carbapenemaseproducing CRAB isolates in urine. Prior to these cases, no previous $\mathrm{CRAB}$ isolates in Colorado had been characterized as carbapenemase-producing organisms. DHMC and the Colorado Department of Public Health and Environment (CDPHE) collaborated to determine epidemiologic and molecular relatedness of the

Author for correspondence: Heather Young MD, Denver Health Medical Center, 601 Broadway, MC 4000, Denver CO 80204. E-mail: Heather.Young2@dhha.org

ADDITIONAL PRESENTATION. This work has been accepted as a poster abstract at the ID Week conference in San Francisco, California, in October 2018.

Cite this article: Young HL. et al. (2018). Collaboration for containment: Detection of OXA-23-like carbapenamase-producing Acinetobacter baumannii in Colorado. Infection Control \& Hospital Epidemiology. 2018, 39, 1273-1274. doi: 10.1017/ice.2018.202 isolates, as well as to investigate healthcare infection control measures.

DHMC is a 555-bed safety net teaching hospital and level 1 trauma center located in Denver, Colorado. DHMC previously reported an MDR-AB outbreak between 2004 and 2005, ${ }^{3}$ and these MDR-AB isolates retained carbapenem susceptibility. Regionally, CRAB is unusual in the Denver metropolitan region, with 2-13 cases reported from sterile body sites and urine per year since 2013.

The CDPHE epidemiologists and DHMC infection preventionists performed surveillance for additional cases that met the case definition. CRAB isolates were defined as those that had a minimum inhibitory concentration (MIC) to at least 1 carbapenem in the intermediate or resistant range. Investigators reviewed medical records for common hospital locations, medical equipment, procedures, and staff members. Infection preventionists observed practices among shared staff members. Pulsed-field gel electrophoresis (PFGE) was performed at the CDPHE laboratory, while antimicrobial susceptibility and carbapenemase testing was performed at the Centers for Disease Control and Prevention (CDC).

The epidemiologic investigation revealed several similarities. Patient 1 was a 59-year-old male with diabetes mellitus and spina bifida, while patient 2 was a 23 -year-old male with lymphangiomatosis and resulting T6 paraplegia. Both patients had neurogenic bladders managed by suprapubic catheters, stage 4 decubitus ulcers, recent surgery, and extensive antibiotic exposure. Neither had recently traveled outside of Colorado nor received a carbapenem in the prior 6 months. CRAB was detected from urine 
culture at the time of hospital admission in both patients and was considered to represent asymptomatic bacteriuria.

One month before identification of the first CRAB, the patients overlapped at DHMC for 8 days on different units. Potential epidemiologic links included radiology and wound care. Both patients had radiographs taken on the same day; one patient traveled to the radiology department, whereas the other had a portable radiograph. Observations of portable radiology technicians revealed consistent and adequate low-level disinfection of equipment and reliable hand hygiene. One wound-care nurse provided care to both patients on the same day. Observations of the wound-care team indicated opportunities to improve hand hygiene prior to donning and after doffing gloves; the use of single-use scissors on multiple patients; and inconsistent cleaning of a mobile device used to photograph open wounds. Discussion with the patients' outpatient providers showed that their suprapubic and wound care supplies were obtained from different companies.

Molecular analyses of the 2 patients' isolates were indistinguishable by PFGE using the restriction enzymes AscI and ApaI. Antimicrobial susceptibility testing revealed that both isolates were susceptible to colistin and resistant to all carbapenems tested. Both harbored OXA-23-like genes according to a Research Use Only assay performed at CDC.

While OXA-23-like enzymes are novel in Colorado, they were first identified in Scotland in 1985 and are the most common carbapenemase enzyme detected worldwide, accounting for $63 \%$ of nosocomial $\mathrm{CRAB}$ in Argentina, $42 \%-100 \%$ in Brazil, 98\% in Colombia, and $55 \%-80 \%$ in Saudi Arabia. ${ }^{4,6,7}$ OXA-23-like enzymes are almost exclusively found in Acinetobacter baumannii and can be encoded by genes located on either a chromosome or plasmid. ${ }^{2,5}$ OXA-23-like enzymes do not require the presence of other resistance mechanisms (eg, porin mutations or efflux pumps) to confer carbapenem resistance. However, when a bacterial strain also carries an efflux pump, the bacteria exhibit higher minimum inhibitory concentration (MIC) to carbapenems as well as resistance to multiple antibiotics, complicating the detection of the gene variant through phenotypic surveillance. ${ }^{5}$

We suspect that the organisms were transmitted during the overlapping hospital admission, although we could not determine where the organism originated or the route of transmission. On the facility level, opportunities to improve hand hygiene and lowlevel disinfection were identified and addressed. The charts were flagged to indicate that the patients harbored an MDR organism and would require contact precautions upon arrival. Infection preventionists notified clinics when upcoming outpatient appointments were detected. The clinics scheduled these patients to be the last of the clinic session when possible to allow for a thorough environmental cleaning after the clinic visit.

On a regional level, CDPHE epidemiologists contacted other healthcare facilities where these patients frequently sought care and encouraged these facilities to also electronically flag medical records and to ensure effective infection control measures. While no further cases of CRAB have been identified to date at DHMC, 1 additional OXA-23-producing CRAB case, without epidemiologic links to the previous 2 patients, has been identified in Colorado since this cluster.

The emergence of previously undetected carbapenemases in Colorado is of great public health concern. Active collaboration and communication between public health and healthcare facilities is critical to halt transmission of novel regional pathogens.

\section{Acknowledgments.}

Financial support. No financial support was provided relevant to this article.

Conflicts of interest. All authors report no conflicts of interest relevant to this article.

\section{References}

1. Munoz-Price LS, Weinstein RA. Acinetobacter infection. $N$ Engl J Med 2008;358:1271-1281.

2. Walsh TR. Clinically significant carbapenemases: an update. Curr Opin Infect Dis 2008;21:367-371.

3. Young LS, Sabel AL, Price CS. Epidemiologic, clinical, and economic evaluation of an outbreak of clonal multidrug-resistant Acinetobacter baumannii infection in a surgical intensive care unit. Infect Control Hosp Epidemiol 2007;28:1247-1254.

4. Paton R, Miles RS, Hood J, Amyes SG, Miles RS, Amyes SG. ARI 1: betalactamase-mediated imipenem resistance in Acinetobacter baumannii. Int $J$ Antimicrob Agent 1993;2:81-87.

5. Evans BA, Amyes SG. OXA beta-lactamases. Clin Microbiol Rev 2014;27:241-263.

6. Labarca JA, Salles MJ, Seas C, Guzman-Blanco M. Carbapenem resistance in Pseudomonas aeruginosa and Acinetobacter baumannii in the nosocomial setting in Latin America. Crit Rev Microbiol 2016;42:276-292.

7. Yezli S, Shibl AM, Memish ZA. The molecular basis of beta-lactamase production in gram-negative bacteria from Saudi Arabia. J Med Microbiol 2015;64:127-136.

\title{
Post-discharge impact of healthcare-associated infections in a developing country: A cohort study
}

\author{
Emília Carolina Oliveira de Souza RN, Sebastião Pires Ferreira Filho MD, MSc, Kasys Meira Gervatauskas MD and \\ Carlos Magno Castelo Branco Fortaleza MD, PhD \\ Department of Tropical Diseases, Botucatu Medical School, São Paulo State University (UNESP), City of Botucatu, São Paulo State, Brazil
}

\footnotetext{
Author for correspondence: Carlos Magno Castelo Branco Fortaleza, Departamento de Doenças Tropicais, Faculdade de Medicina de Botucatu, Distrito de Rubião Júnior, S/N, City of Botucatu, São Paulo State Brazil 18618-970. E-mail: cmfortaleza@uol.com.br

Cite this article: Madden GR, et al. (2018). Post-discharge impact of healthcareassociated infections in a developing country: A cohort study. Infection Control \& Hospital Epidemiology 2018, 39, 1274-1276. doi: 10.1017/ice.2018.201
}

To the Editor - The impact of healthcare-associated infections (HCAIs) on in-hospital mortality, morbidity, length-of-stay, and costs has been extensively reported. ${ }^{1,2}$ However, few studies have focused on the follow-up of HCAI-affected subjects after 